\title{
Spin-Resolved Photoemission from Xe-Adlayers on Pd(111)
}

\author{
B. Kessler, N. Müller, B. Schmiedeskamp, B. Vogt and U. Heinzmann \\ Universität Bielefeld, Fakultät für Physik, D-4800 Bielefeld 1, F.R.G.; and Fritz-Haber-Institut der MPG, D-1000 Berlin 33, F.R.G.
}

Received July 16, 1989; accepted January 26, 1990

\begin{abstract}
Xe-adsorbates on $\mathrm{Pd}(111)$ in the coverage region from one layer up to a $\mathrm{Xe}(111)$ crystal with first layer Xe-Xe distances comparable to bulk Xe are studied by spin-resolved photoemission. Normally incident circularly polarized synchrotron radiation from BESSY (Berlin) is used and normally emitted electrons are analyzed. No dispersion with photon energy can be found for a system of two Xe-layers. The spin-polarization component measured along the direction of light points to a changing symmetry of the final states. Their symmetry is atom-like for a one-layer system and bulk-like for two and more layers. The initial state symmetry is examined by excitation and decay of excitonic states and is found to be the same for two Xe-layers as for one single Xe-layer. The degeneracy of the valence levels connected with the high symmetry of the $\Gamma$-point in bulk Xe can be observed for a system of much more than $5 \mathrm{Xe}$-layers thickness.
\end{abstract}

Xe-adsorbates have been studied by different techniques [1]. Spin-resolved photoelectron spectroscopy of Xe-adsorbates using circularly polarized synchrotron radiation has been shown to be a suitable method to get information beyond intensity spectroscopy [2-6]: The lateral Xe-Xe interaction has been found to dominate the splitting of the $\left|m_{j}\right|$-sublevels of the $p_{3 / 2}$-state $[5,6,23]$. The bandstructure of bulk Xe along $\Lambda$ has been elaborated by symmetry-resolved bandmapping using relativistic dipole selection rules [7,8]. An emission process via the excitation and decay of an excitonic state has been found at photon energies below the threshold of direct photoemission for Xe-coverages from one Xe-layer to a Xe-crystal $[3,5]$.

An interesting question is to understand the development of the properties of a three-dimensional Xe-crystal when two-dimensional Xe-layers are stacked. Layer by layer growth of Xe-adsorbates has been intensively studied by spectroscopy of photoelectron intensities. The layer dependent photoemission-peak shifts observed in going from a single adsorbate layer to multilayers have been discussed controversely $[9,10]$. Recent experiments with $\mathrm{Ar}, \mathrm{Kr}$ and $\mathrm{Xe}$ on $\mathrm{Pb}(111)$ demonstrate that these peak shifts are due to the change of the relaxation of the final-state hole [11]. This interpretation was firstly proposed in Ref. [12] and was used in Ref. [13] to interpret layer-dependent photoemission intensity-spectra from Xe/Al(111). The spectra of the twolayer system have been found to be the sum of two one-layer spectra shifted against each other. The first hints for bulk bands have appeared at about 2.5 layers.

The aim of our experiment is to study the onset of effects of the third dimension in the layer by layer growth of $\mathrm{Xe} / \mathrm{Pd}(111)$ by spin-resolved photoelectron spectroscopy. The measurements are performed at the $6.5 \mathrm{~m}$ NI-monochromator [14] for circularly polarized radiation at BESSY in Berlin. The experimental setup has been previously described [15]. The circularly polarized $(90 \pm 3 \%)$ synchrotron radiation with energies from 7.8 to $17 \mathrm{eV}$ hits normally the target.
The normally emitted photoelectrons are energy analyzed by a simulated hemispherical-field spectrometer [16]. For electrons with a kinetic energy of $1 \mathrm{eV}$ the angular resolution is $\pm 7.5 \mathrm{deg}[17]$. The overall resolution (photons plus electrons) is better than $200 \mathrm{meV}$. The spin-polarization component $P$ in the direction of light incidence is measured by Mott scattering. Positive $P$ means electron-spin polarization parallel to the photon spin [18]. The experimental results are displayed in the form of partial intensities $I_{+}$and $I_{-}$giving the parts of the emitted electrons with expectation value of the polarization operator +1 or -1 , respectively. $I_{+}$and $I_{-}$result from the total electron intensity $I_{0}$ and the spin polarization $P$ by:

$I_{+}=0.5 I_{0}(1+P) \quad I_{-}=0.5 I_{0}(1-P)$.

The Pd(111)-substrate crystal can be cooled by liquid helium and is cleaned by Ne-sputtering, heating in oxygen at about $800 \mathrm{~K}$ and flashing to about $1300 \mathrm{~K}$. The crystal surface is characterized by LEED, by Auger-spectroscopy and concerning $\mathrm{C}$-contamination by cycles of oxygen adsorption and CO-desorption spectroscopy [19].

The Xe-adsorbate is prepared by cooling the substrate down to temperatures between 100 and $40 \mathrm{~K}$ and subsequent $\mathrm{Xe}$ deposition via a gas doser. It is controlled by photoemission-peak shifts [9-13] and by LEED. Always a layer with a LEED-pattern showing a hexagonal structure with two domains rotated by $26 \pm 2 \mathrm{deg}$ with respect to the substrate has been prepared as a first layer. The $\mathrm{Xe}-\mathrm{Xe}$ distance within this first layer turns out to be $4.25 \pm 0.15 \AA$ and is thus comparable to the bulk Xe-Xe distance of $4.37 \AA$ [20]. A comparable structure with a $\mathrm{Xe}-\mathrm{Xe}$ distance of $4.33 \pm 0.03 \AA$ and a rotation by 26.7 deg has been found for single Xe-layers on nearly defect free $\operatorname{Pt}(111)$ and has been interpreted as a high-order commensurate phase: each eleventh Xe-atom is located at a high symmetry site [21, 22]. As the nearest-neighbor distances of $\mathrm{Pd}$ and $\mathrm{Pt}$ are nearly identical $(2.75 \AA$ for $\mathrm{Pd}$ and $2.77 \AA$ for $\mathrm{Pt}$ [20]), we suppose that the Xe-layer on $\mathrm{Pd}(111)$ showing the $26 \mathrm{deg}$ rotation has to be interpreted in a similar way. Due to the better agreement with the bulk Xe-Xe distance this first layer structure seems to be a more suitable basis for a layer by layer growth of a Xe-crystal than the more compressed and more buckled $\mathrm{Xe}(\sqrt{7} \times \sqrt{7}) \mathrm{R} 19,2^{\circ} / \mathrm{Pd}(111)$ layer [23]. The Xe-atoms of this adsorbate layer show a distance of $4.2 \pm 0.05 \AA$ in the projection to the surface plane which is a lower limit for the $\mathrm{Xe}-\mathrm{Xe}$ distance in the less compressed first layer of the adsorbates used in the present work. The \pm 26 deg rotation of the two Xe-domains has not to be considered because circularly polarized light with normal incidence and normal photoelectron emission has been used.

Figure 1 shows the photoelectron spectra of $\mathrm{Xe} / \mathrm{Pd}(111)$ for a photon energy of $h v=12.5 \mathrm{eV}$ and various Xe-coverages. 


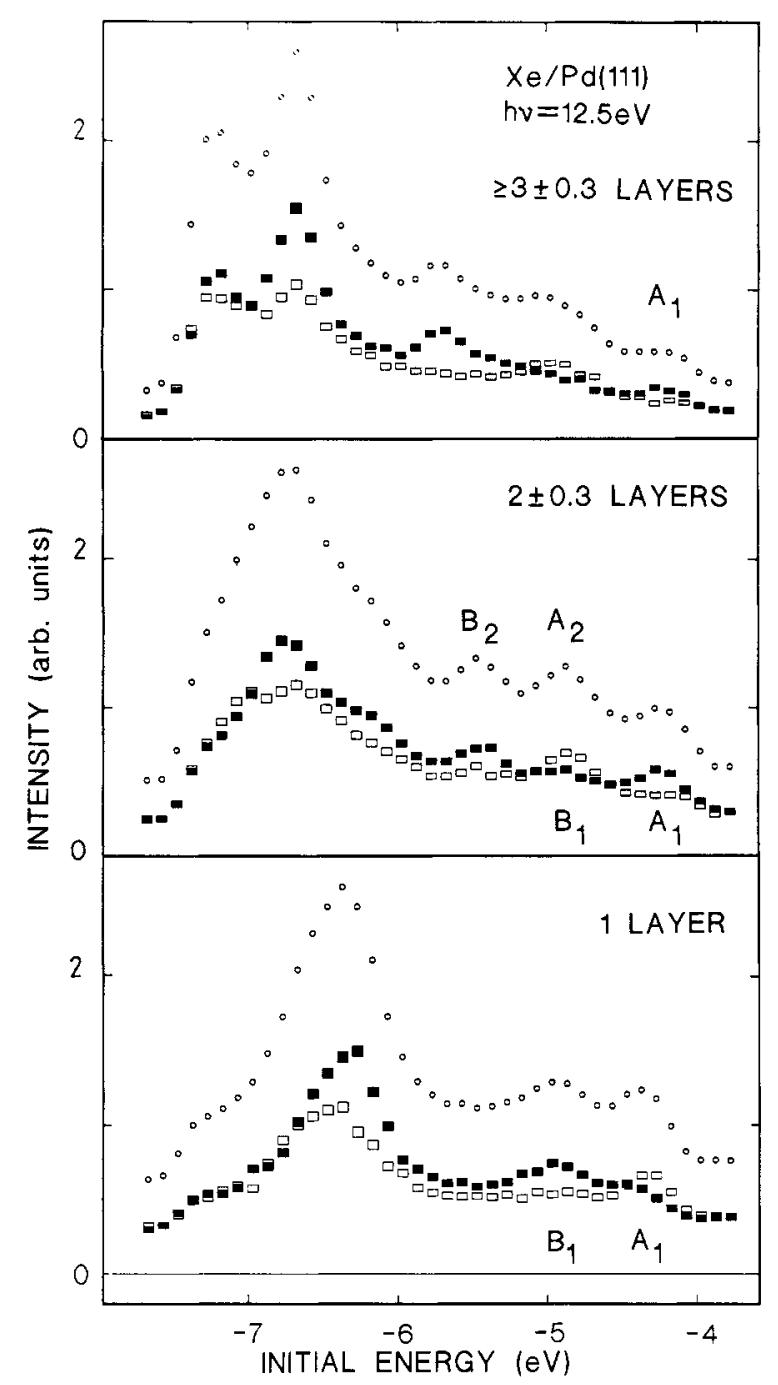

Fig. 1. Spin-resolved photoemission spectra from $\mathrm{Xe} / \mathrm{Pd}(111)$ at different coverages and $h v=12.5 \mathrm{eV}$. (O: total intensity $I_{0} ; \mathbf{\square}$ : partial intensity $I_{+}$; $\square$ : partial intensity $I_{-}$); the heights of the $I_{+} / I_{-}$-symbols represent the uncertainties given by the errors of the total intensity and the polarization. The intensities are normalized to equal height of the largest maxima. The energy refers to the Fermi level of the substrate. The peak assignments are given in the text.

The one Xe-layer spectrum at the bottom of the figure shows three peaks in the total intensities. They correspond to the $p_{1 / 2^{-}}$and the two $\left|m_{j}\right|$-splitted $p_{3 / 2}$-hole states of the Xe-adsorbate which can be seen by comparing the spindependent partial intensities $I_{+}$and $I_{-}$with the results of Ref. [6] $\left(p_{1 / 2}\right.$ : peak in $I_{+}, p_{3 / 2}\left|m_{j}\right|=\frac{1}{2}$ : peak in $I_{+}, p_{3 / 2}\left|m_{j}\right|=$ $\frac{3}{2}$ : peak in $\left.I_{-}\right)$. The large $I_{-}$-part in the $p_{1 / 2}$-peak at about $-6.4 \mathrm{eV}$ might be due to a density of states feature and will be not discussed further. We will focus on the $\left(p_{3 / 2}\right.$, $\left.\left|m_{j}\right|=\frac{3}{2}\right)$-peak at $-4.3 \mathrm{eV}$ and the $\left(p_{3 / 2},\left|m_{j}\right|=\frac{1}{2}\right)$-peak at $-4.9 \mathrm{eV}$ denoted as $\mathrm{A}_{1}$ and $\mathrm{B}_{1}$, respectively. When going to the spectrum of $2 \pm 0.3 \mathrm{Xe}$-layers (Fig. 1, middle panel) the corresponding transitions starting from Xe-atoms of the second layer (denoted by $A_{2}$ and $B_{2}$ ) occur at lower initial state energies. The preferential spin direction in the peaks at $-4.9 \mathrm{eV}$ and $-5.5 \mathrm{eV}$ seems to be dominated by the transitions $A_{2}$ and $B_{2}$, respectively. A surprising effect is the change of the spin polarization measured in the peak $A_{1}$ (originating from the first layer) from dominating partial intensity $I_{-}$to dominating partial intensity $I_{+}$when a second layer is adsorbed. If the coverage is increased to more than three Xe-layers (Fig. 1, top panel) peak $\mathrm{A}_{1}$ still shows positive

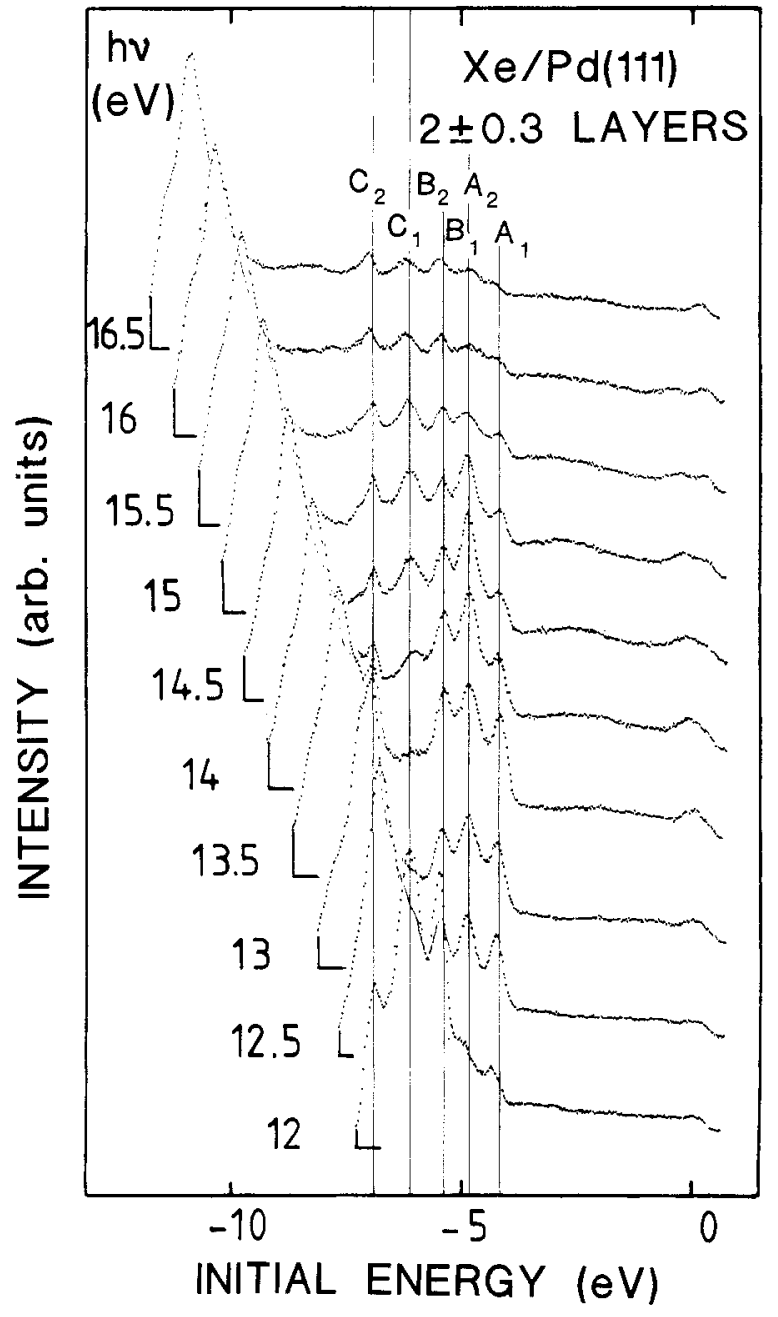

Fig. 2. Total photoemission spectra for a system of $2 \pm 0.3$ Xe-layers on $\mathrm{Pd}(111)$ at varied photon energies. The intensities are normalized to equal height of the largest maxima. (Peak assignments see text.)

spin polarization though the intensity decreases relative to the other peaks. The intensity decrease can be understood by the limited free path of the electrons which are created in the first Xe-layer and which have to cross another two Xe-layers before leaving the adsorbate. The positive spin polarization in peak $A_{1}$ at a coverage of 2 Xe-layers can not be understood in a model assuming photoemission from two independent layers with identical symmetry of the electronic states.

Three dimensional systems typically show dispersion of photoemission-intensity peaks when the photon energy is varied (e.g. Ref. [7]). Figure 2 shows the photoelectronintensity spectra for a system of two Xe-layers on Pd(111) at different photon energies between 12 and $16.5 \mathrm{eV}$. The large peak at constant kinetic energy near the vacuum edge might be due to a density of states feature [7]. Here this peak will not be discussed in detail. Near $E_{F}$ some weak features of Pd are visible. The peaks between -4 and $-7 \mathrm{eV}$ correspond to photoelectrons from the adsorbate. Going from high to low initial energy they can be asssigned as $A_{1}, B_{1} / A_{2}, B_{2}, C_{1}, C_{2}$ $\left(C_{1}\right.$ and $C_{2}$ denote emission from the $p_{1 / 2}$-states of the first and second Xe-layer, respectively). The initial energies do not significantly vary with photon energy: we can not find dispersion for the two Xe-layer system. This behaviour is not very surprising remembering other results from systems of two Xe-layers [9-13].

The symmetries of the initial states can be analyzed by constant final state spectroscopy (CFS): The total intensity $I_{0}$ 


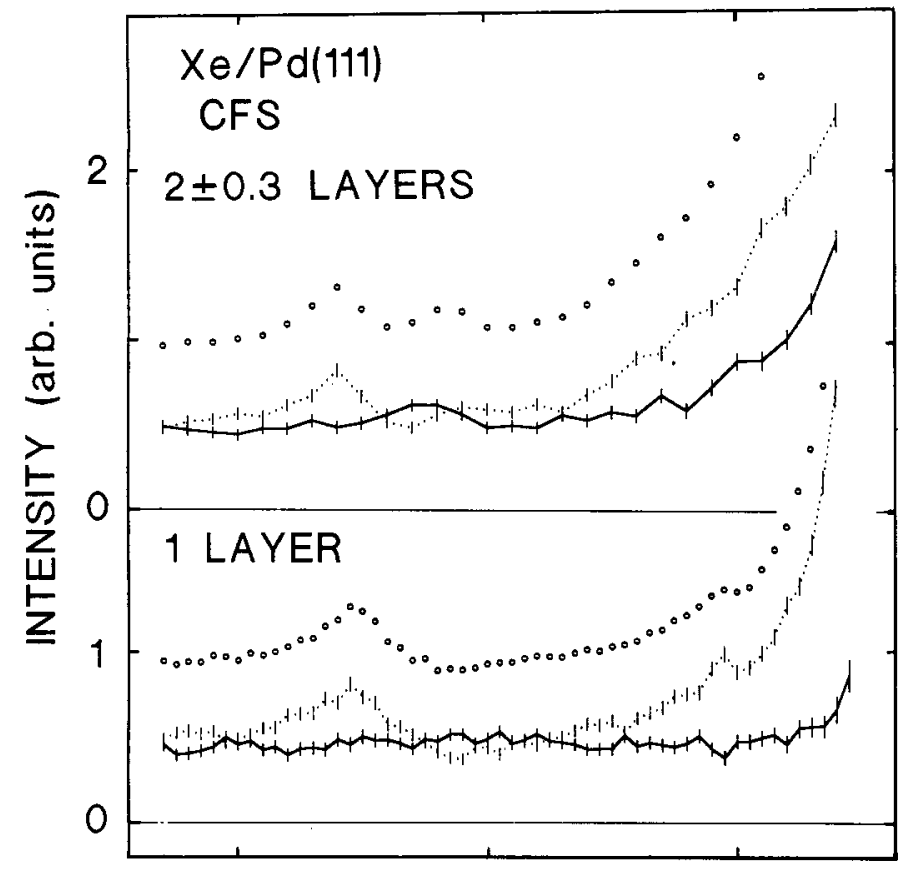

8 9

PHOTON ENERGY (eV)

Fig. 3. Spin-resolved constant final state spectra at final state energy $E_{\mathrm{f}} \approx 0.4 \mathrm{eV}$ with photon energies below the threshold for direct photoemission showing emission via excitonic states at different coverages. $(0$ : $\left.I_{0}, I_{+}, \cdots \cdot I_{-}\right)$. The intensity scales are not comparable.

and the spin polarization $P$ of electrons with a constant kinetic energy of about $0.4 \mathrm{eV}$ are measured under variation of photon energy starting at photon energies below the onset of direct photoemission. Figure 3 shows these CFS-spectra for one Xe-layer (bottom of Fig. 3) and for $2 \pm 0.3$ Xe-layers (top of Fig. 3) on Pd(111). Above a photon energy of $9 \mathrm{eV}$ the intensity increases dramatically due to the onset of direct photoemission. We will not discuss this region. The feature of main interest in the context of this paper is the peak in the partial intensity $I_{-}$at a photon energy of about $8.2 \mathrm{eV}$ which results from the decay of an excited excitonic state of the adsorbate $[3,5]$. The exciton belongs to an excited state with symmetry $\left[5 p^{5}\left({ }^{2} P_{3 / 2}\left|M_{J}\right|=\frac{3}{2}\right) 6 s\right]_{1}^{0}[5]$. The core of this state has the same symmetry as the initial hole states corresponding to the $A_{1}-, A_{2}$-peaks in direct photoemission. In contrast to the results from direct photoemission the peak in the CFSspectra of Fig. 3 does not change the sign of spin polarization when going from one Xe-layer to two Xe-layers on Pd(111). We conclude therefore that the change of sign observed in direct photoemission is not induced by a change in the symmetry of the initial state.

The lack of dispersion for a system of two Xe-layers under variation of the photon energy allows to study the change of the preferential spin direction of peak $A_{1}$ by constant initial state spectroscopy (CIS). Figure 4 shows a CIS-spectrum for two Xe-layers with an initial energy of $-4.3 \mathrm{eV}$ corresponding to the $A_{1}$ initial state. The electron intensities measured are not corrected for the photon flux of the monochromator as the photon flux has been measured to be nearly constant for photon energies between 11 and $15 \mathrm{eV}$ [14]. The decrease of electron intensity at photon energies higher than $15 \mathrm{eV}$ may be caused by the decreasing flux having a minimum at

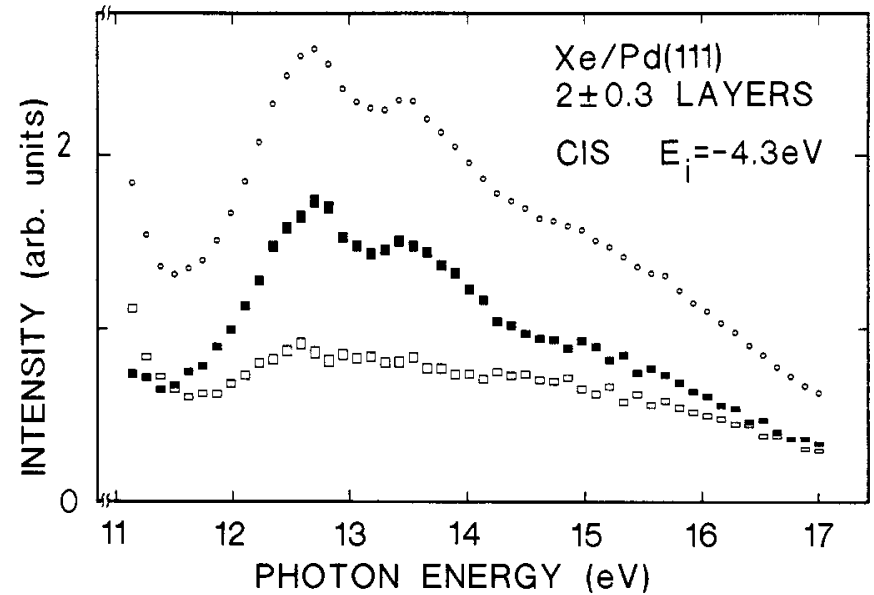

Fig. 4. Spin-resolved constant initial state spectrum at initial state energy of $-4.3 \mathrm{eV}$ for $2 \pm 0.3$ Xe-layers. (O: $I_{0}, \boldsymbol{\square}: I_{+}, \square: I_{-}$).

about $20 \mathrm{eV}$. The partial intensity $I_{+}$dominates for all photon energies measured above about $11.5 \mathrm{eV}$. This is in accordance with the results from Fig. 1 at $h v=12.5 \mathrm{eV}$ where the peak $\mathrm{A}_{1}$ is caused by the partial intensity $I_{+}$for a system of two $\mathrm{Xe}$-layers. However at photon energies below about $11.5 \mathrm{eV}$ the partial intensity $I_{-}$dominates in the peak $\mathrm{A}_{1}$. We conclude that the symmetry of the final state involved changes and this change causes the positive sign of spin polarization for photon energies above $11.5 \mathrm{eV}$. The maxima in the partial intensity $I_{+}$at about 12.7 and $13.4 \mathrm{eV}$ may be induced by a high density of final states.

The spin-polarization behavior of peak $A_{1}$ can be explained by a comparison with the bandstructure of a Xe-crystal in the $\Lambda$-direction [7]. Considering the screening effects induced by the substrate which are highest for the first Xe-layer the $A_{1}$ initial state lies $0.7 \mathrm{eV}$ above the valence band maximum (VBM) of solid Xe. This initial energy shows no dispersion, it is flat between $\Gamma$ and $L$. The symmetry of the $A_{1}$ initial state is purely $x, y(-z$ is the direction of the surface normal) [24] which is mostly comparable to a band which transforms according to $\Lambda_{4.5}^{3}$ [25]. From the dipole selection rules in the $\Lambda$-direction of f.c.c. and b.c.c. crystals [8] it follows that negative and positive spin polarization characterizes transitions from states with symmetry $\Lambda_{4,5}^{3}$ into empty states of symmetry $\Lambda_{6}^{1}$ and $\Lambda_{6}^{3}$, respectively. Regarding to the bandstructure given in Ref. [7] the lowest lying empty band along $\Lambda$ transforms as $\Lambda_{6}^{1}$. Going to higher energies an energy gap between 11 and $13 \mathrm{eV}$ and two flat hybridized bands with $\Lambda_{6}^{3} / \Lambda_{6}^{1}$-symmetry follow at about 13.8 and $14.3 \mathrm{eV}$ above VBM. A CIS-measurement with the initial energy of an ideal flat $\Lambda_{4,5}^{3}$-band located $0.7 \mathrm{eV}$ above VBM should therefore result in negative polarized photoelectrons up to a photon energy of $10.3 \mathrm{eV}$ and positive polarized photo-electrons above $h v=12.3 \mathrm{eV}$ with maximum intensities at 13.1 and $13.6 \mathrm{eV}$. For photon energies between about 10.3 and $12.3 \mathrm{eV}$ the gap should induce a zone of nearly vanishing electron intensity. A comparison with the CIS-spectrum of Fig. 4 shows a quite good agreement though the gap is strongly weakened and the energies do not coincide exactly. We conclude therefore that the spin-polarization measured for two $\mathrm{Xe}$-layers can roughly be explained by assuming empty states with symmetries corresponding to those of bulk Xe.

An important characteristic of the bandstructure of a three dimensional Xe-crystal is the degeneracy of the two topmost 


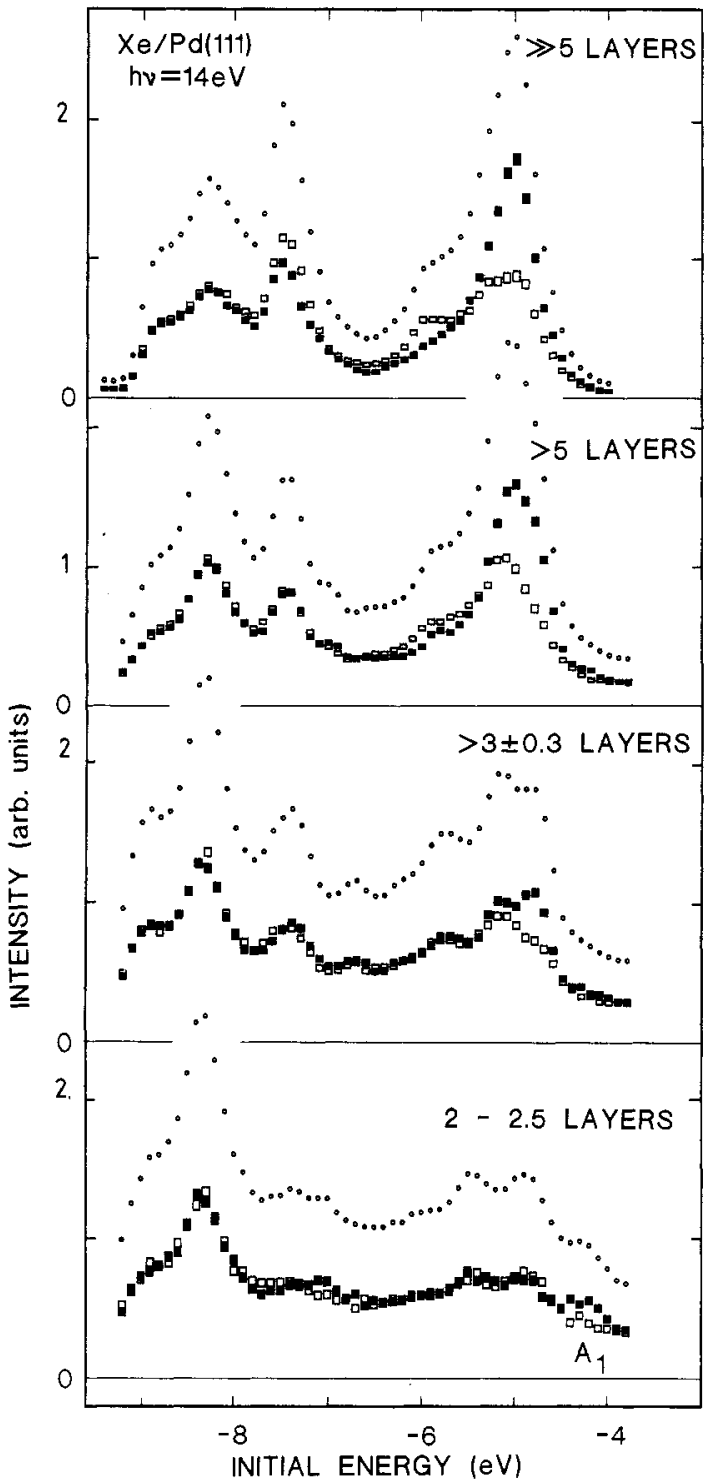

Fig. 5. Spin-resolved photoemission spectra for $\mathrm{Xe} / \mathrm{Pd}(11 \mathrm{l})$ at different coverages and $h v=14 \mathrm{eV} .\left(0: I_{0}, \boldsymbol{\omega}: I_{+}, \square: I_{-}\right.$, peak assignments see text).

valence levels at the $\Gamma$-point due to the high symmetry of this point. With a Xe-crystal electrons from the $\Gamma$-point can be observed at a photon energy of $14 \mathrm{eV}$. Figure 5 shows photoemission spectra at $h v=14 \mathrm{eV}$ at various Xe-coverages on a $\operatorname{Pd}(111)$-substrate. We will concentrate on the initial energies from about -4 to $-5 \mathrm{eV}$. At low coverage (2-2.5 Xe-layers, Fig. 5, bottom panel) the total intensity shows discrete peaks. At $-4.3 \mathrm{eV}$ peak $\mathrm{A}_{1}$ can be identified due to its positive spin polarization. With increasing Xe-coverage $(>3 \pm 0.3$ layers and $>5$ layers, Fig. 5) an asymmetric peak at about $-5 \mathrm{eV}$ is built up in the total intensity by one peak in the partial intensity $I_{+}$and one slightly shifted peak in the partial intensity $I_{-}$. For a system of more than 5 Xe-layers thickness the $I_{+}$-peak and the $I_{-}$-peak still show a splitting of at least $0.1 \mathrm{eV}$. When the coverage is further increased $(\gg 5 \mathrm{Xe}-$ layers, Fig. 5, upper panel) we get a three dimensional crystal and the splitting vanishes. The spectrum equals those obtained from $\mathrm{Xe}(111)$ on $\operatorname{Ir}(111)$ and $\operatorname{Pt}(111)$ [7]. The $I_{+}$-peak and the $I_{-}$-peak at about $-5 \mathrm{eV}$ characterize the VBM of the Xe-crystal where the two topmost valence bands are degenerated. Assuming a transition in an empty band of $\Lambda_{6}^{3} / \Lambda_{6}^{1}$-symmetry the $I_{+}$-peak and the $I_{-}$-peak originate from the $\Lambda_{4,5}^{3}$ and the $\Lambda_{6}^{1}$ initial bands, respectively. We conclude that for $5 \mathrm{Xe}$-layers the band structure of the Xe-crystal is not yet developed which might be due to the lack of degeneracy or to an energy shift of the levels involved.

Summarizing spin resolved photoemission data in normal incidence of circularly polarized synchrotron radiation and normal electron emission from Xe-adsorbates on $\mathrm{Pd}(111)$ are presented. The hexagonal adlayer with a Xe-Xe distance comparable to that of bulk Xe growing in two domains rotated by $26 \pm 3 \mathrm{deg}$ is supposed to be high-order commensurate and to be an ideal first layer for growing of a Xe-crystal. The symmetry of the initial state corresponding to the atomic $p_{3 / 2}\left|m_{j}\right|=\frac{3}{2}$ level is the same for one Xe-layer and for two Xe-layers as can be seen by excitation and decay of excitonic states. From spin-resolved photoemission it results that in a system of two Xe-layers the empty electronic states normal to the layer show symmetries comparable to bulk Xe along $\Lambda\left(\Lambda_{6}^{1}\right.$ and $\left.\Lambda_{6}^{3} / \Lambda_{6}^{1}\right)$. the gaps are weakened. The completely developed bandstructure of solid Xe near $\Gamma$ can only be observed for a system of much more that 5 Xe-layers.

\section{Acknowledgements}

We would like to thank G. Schönhense for stimulating and fruitful discussions. We also express our thanks to the staff of BESSY for their continuous help. The work was supported by the Bundesministerium für Forschung und Technologie (05 331 and $431 \mathrm{Ax}$ ).

\section{References}

1. Ordering in Two Dimensions (Edited by S. K. Sinha), North Holland, New York (1980).

2. Heinzmann, U., Physica Scripta T17, 77 (1987)

3. Schönhense, G., Eyers, A. and Heinzmann, U., Phys. Rev. Lett. 56, 512 (1986); Schönhense, G., Kessler, B., Müller, N., Schmiedeskamp, B., and Heinzmann, U., Physica Scripta 35, 541 (1987).

4. Heinzmann, U. and Schönhense, G., Polarized Electrons at Surfaces (Edited by R. Feder), Ch. XI, p. 467ff., World Scientific, Singapore (1985).

5. Schönhense, G., Appl. Phys. A41, 39 (1986).

6. Schönhense, G., Eyers, A., Friess, U., Schäfers, F. and Heinzmann, U., Phys. Rev. Lett. 54, 547 (1985).

7. Kessler, B., Eyers, A., Horn, K., Müller, N., Schmiedeskamp, B., Schönhense, G. and Heinzmann, U., Phys. Rev. Lett. 59, 331 (1987).

8. Wöhlecke, M. and Borstel, G., Optical Orientation (Edited by F. Meier and B. P. Zakharchenya), p. 423. North Holland, Amsterdam (1984).

9. Steinberger, I. T. and Wandelt, K., Phys. Rev. Lett. 58, 2494 (1987) (and references therein).

10. Kaindl, G. and Mandel, T., Phys. Rev. Lett. 59, 2238 (1987) (and references therein).

11. Jacobi, K., Phys. Rev. B38, 5869 (1988).

12. Kaindl, G., Chiang, T. -C., Eastman, D. E. and Himpsel, F. J., Phys. Rev. Lett. 45, 1808 (1980)

13. Mandel, T., Kaindl, G., Domke, M., Fischer, W. and Schneider, W. K., Phys. Rev. Lett. 55, 1638 (1985).

14. Schäfers, F., Peatman, W., Eyers, A., Heckenkamp, Ch, Schönhense, G. and Heinzmann, U., Rev. Sci. Instrum. 57, 1032 (1986).

15. Eyers, A., Schäfers, F., Schönhense, G., Heinzmann, U., Oepen, H. P., Hünlich, K., Kirschner, J, and Borstel, G., Phys. Rev. Lett. 52, 1559 (1984).

16. Jost, K., J. Phys. E12, 1001, 1006 (1979).

17. Due to an acceleration between target and spectrometer entrance aperture the angular resolution depends on the kinetic energy of the emitted electrons. Considering an inner potential of about $-4 \mathrm{eV}$ [Scheffler, M., Horn, K., Bradshaw, A. M. and Kambe, K., Surf. Sci. 80, 69 (1979)] these emission angles correspond to much smaller angles inside the adsorbate. 
18. This convention is the convention used in photoemission from free atoms. It is opposite to that used in Ref. [8].

19. Wandelt, K., Private communication.

20. Wyckoff, R. W. G., Crystal Structures, 2nd ed., Vol. II. Interscience, New York (1964).

21. Poelsema, B., Verheij, L. K., and Comsa, G., Surf. Sci. 152/153, 851 (1985).

22. Kern, K., David, R., Palmer, R. L. and Comsa, G., Appl. Phys. A41, 91 (1986).
23. Vogt, B., Kessler, B., Müller, N., Schmiedeskamp, B., Schönhense, G. and Heinzmann, U., To be published.

24. Meier, F. and Pescia, D., Optical Orientation (Edited by F. Meier and B. P. Zakharchenya), p. 295 ff, North Holland, Amsterdam (1984).

25. Koster, G. F., Dimmock, J. O., Wheeler, R. G. and Statz, H., Properties of the Thirty-Two Point Groups, 3rd ed. MIT Press, Cambridge, MA (1969). 